\section{P063/011 INHIBITION OF ARGINASE-1 EXPRESSION BY THE TRANSCRIPTION FACTOR FRA-1 IN MACROPHAGES EXACERBATES RHEUMATOID ARTHRITIS INFLAMMATION}

${ }^{1} \mathrm{~N}$ Hannemann*, 'S Cao, ${ }^{1} \mathrm{~A}$ Schnelzer, ${ }^{2} \mathrm{~J} J o r d a n,{ }^{3} \mathrm{M}$ Eberhardt, ${ }^{4} \mathrm{U}$ Schleicher, ${ }^{5} \mathrm{~S}$ Uebe, ${ }^{6} \mathrm{~A}$ Ekici, ${ }^{1} \mathrm{~J}$ Rech, ${ }^{2} \mathrm{~T}$ Bäuerle, ${ }^{4} \mathrm{C}$ Bogdan, ${ }^{3} \mathrm{~J}$ Vera, ${ }^{1} \mathrm{G}$ Schett, ${ }^{1} \mathrm{~A}$ Bozec. ${ }^{1}$ Department of Internal Medicine 3; ${ }^{2}$ Institute of Radiology, Preclinical Imaging Platform Erlangen (PIPE); ${ }^{3}$ Department of Dermatology; ${ }^{4}$ Institute of Microbiology - Clinical Microbiology; ${ }^{5}$ Institute of Human Genetics Universitätsklinikum Erlangen; ${ }^{6}$ Institute of Human Genetics, erlangen, Germany

\subsection{6/annrheumdis-2018-EWRR2019.53}

Career situation of first and presenting author Student for a master or a $\mathrm{PhD}$.

Introduction The activator protein (AP)-1 transcription factor family, especially its subfamily of FOS proteins (cFos, FosB, Fra-1 and Fra-2) are associated to the regulatory network of macrophage responses. Moreover, it is well known that macrophages are central player during rheumatoid arthritis (RA).

Objectives This study aims to delineate the role of Fra-1 in macrophages during the acute destructive inflammatory phase of RA.

Methods Therefore, we applied the serum-induced arthritis (K/ BxN) model to Fra-1 deficient mice controlled by the $\mathrm{Mx} 1$ promoter $\left(\right.$ Fra- $\left.1^{\Delta \mathrm{Mx}}\right)$ or the LysM promoter (Fra- $1^{\Delta \mathrm{LysM}}$ ). Moreover, we performed in vitro analyses of macrophage polarization in wildtype and Fra-1 deficient macrophages, as well as micro-arrays and ChIP sequencing analyses to delineate Fra-1 targets in activated macrophages. We completed our analysis by studying Fra-1 expression in RA patients' synovium.

Results Fra-1 mutant mice had decreased arthritis severity compared to their littermate wildtype mice. The alleviated arthritis was accompanied to increased arginase-1 (Arg1) expression and activity in the joints, suggesting that its anti-inflammatory features milder RA inflammation. Sorting of immune cell populations revealed macrophages as the major source of Arg1, which was increased in Fra-1 mutant mice. Mechanistically, Fra-1 transcriptionally inhibited Arg1 expression in macrophages. Moreover, inhibition of Arginase in Fra-1 mutant mice restored a full blunt inflammatory RA response and the supplementation of mice with l-arginine, leading to increased arginase activity in the joint, is sufficient to milder arthritis. Synovium histological sections from RA patients showed a correlation between Arg1, Fra-1 and the DAS28 score, confirming that increased Arg1 activity is of benefit also for human inflammatory joint disease.

Conclusions Our data show for the first time that Fra-1 is a pivot between pro- and anti-inflammatory macrophage. By inhibiting Arg1 activity, Fra-1 exacerbates RA inflammation and joint destruction.

Disclosure of Interest None declared.

\section{P065 REGULATION OF JOINT DESTRUCTION BY ACTIVIN A IN RHEUMATOID ARTHRITIS}

V Kracke*, M Fennen, J Intemann, E Werbenko, D de Gorter, P Paruzel, T Pap, B Dankbar. Institute of Musculosceletal Medicine, Münster, Germany

10.1136/annrheumdis-2018-EWRR2019.54

Career situation of first and presenting author Student for a master or a $\mathrm{PhD}$.
Introduction Activins and inhibins belong to the transforming growth factor $\beta$ family. Activins are disulphide-linked homoor heterodimers consisting of two inhibin $\beta$ chains $(\beta A, \beta B)$ that are expressed in many cell types. However, activin A ( $\beta A$ $\beta A)$ is the only activin that is expressed in bone and cartilage. Moreover, activin A has been demonstrated not only to stimulate receptor activator of NF- $\kappa \mathrm{B}$ ligand (RANKL)-induced osteoclast (OC) differentiation but also to inhibit osteoblast differentiation.

Objectives Here we investigate the impact of activin A on joint destruction in rheumatoid arthritis.

Methods Synovial tissue samples from rheumatoid arthritis (RA) and osteoarthritis (OA) patients were analysed by immunohistochemical staining. For in vitro experiments, synovial fibroblasts (FLS) were isolated from hind paws of WT mice. Effects of cytokines on the secretion of activin A by mouse FLS were evaluated by ELISA. Bone marrow-derived macrophages (BMM) were isolated from femurs and tibias of WT mice and differentiated into osteoclasts in the presence of macrophage colony-stimulating factor (M-CSF) and RANKL with or without activin A. OC differentiation was characterised by TRAP staining. Resorption activity was determined by quantification of osteoclast-mediated pit formation on a calcium phosphate-coated plate. Furthermore, osteoclast-specific gene expression as well as the activation of SMAD2 in BMMs, OCs and FLS were analysed by immunoblotting. The interaction of phospho-SMAD2 with NFATc1 was evaluated by co-immunoprecipitation using Dynabeads.

Results We demonstrate that activin A is highly abundant in the synovium of RA but not of OA patients. In vitro, activin A secretion by FLS was strongly enhanced by pro-inflammatory cytokines. Furthermore, activin A strongly enhanced the RANKL-mediated differentiation of BMMs into mature OCs, reflected by a significantly increased OC number and OC size. Moreover, concomitant administration of activin A led to a significant increase of the total resorption area as well as resorption area per pit, indicating an increased activity of individual OCs. Furthermore, activin A enhanced the RANKLinduced expression OC differentiation markers, but alone was not able to induce OC differentiation. Analyses of signaling pathways revealed that activin $A$ induce the activation of SMAD2 in BMMs and OCs. Finally, upon co-stimulation with RANKL, activin A resulted in an increased interaction between activated SMAD2 and NFATc1.

Conclusions The data strongly suggest that increased expression of activin A in the arthritic joint is associated with enhanced osteoclast formation, promoting joint destruction in rheumatoid arthritis.

Disclosure of Interest None declared.

\section{\begin{tabular}{l|l} 
P066 & MIR-342-3P PROMOTES CELL SURVIVAL AND
\end{tabular} MOTILITY OF OSTEOCLAST PRECURSORS}

${ }^{1,2,3} \mathrm{C}$ Lozano*, 1,3V Estibals, ${ }^{1,3}$ I Duroux-Richard, 1,2,3 F Apparailly. ${ }^{1}$ U1183-IRMB, INSERM; ${ }^{2}$ CHU Montpellier; ${ }^{3}$ University Montpellier, Montpellier, France

\subsection{6/annrheumdis-2018-EWRR2019.55}

Career situation of first and presenting author Young investigator.

Introduction Chronic inflammatory joint disorders are associated with bone destruction by osteoclasts (OC), which derive from myeloid precursors. Recent findings reveal that $\mathrm{OC}$ are 
not only professional bone-resorbing cells but also directly involved in controlling inflammatory responses. Depending on the pathophysiological context, OC can polarize the immune response towards tolerance or inflammation. Aiming at identifying key regulators of inflammatory OC functions, we have defined a miRNA-based signature, which includes miR-342-3p. MiRNAs are key regulators of gene expression that control cellular processes, including osteoclastogenesis, and few miRNAs have been described in the differentiation of myeloid precursors into mature OC.

Objectives To study the role of miR-342-3p in inflammatory OC.

Methods OC were derived from the murine monocyte RAW264.7 cells. The expression levels of miR-342-3p and OC-specific genes were monitored by qRT-PCR. RAW264.7 cells were transfected with either miR-342-3p mimics, neutralizing molecules or control miRNAs. Cell survival and proliferation were assessed at 48 hour after RANKL incubation using quantification of the caspase3-7 activity, ATP production and BrDU incorporation. Motility of OC precursors was monitored using time-laps during the course of OC differentiation. The $\mathrm{K} / \mathrm{BxN}$ serum-transfer arthritis (STA) model was performed in 8 weeks old $\mathrm{C} 57 \mathrm{BL} / 6$ males and bone marrow was flushed. Primary OC were generated from either total bone marrow or sorted $\mathrm{CD}_{11} \mathrm{~b}^{+}$and $\mathrm{CD} 11 \mathrm{c}^{+}$cell subsets of healthy and arthritic mice.

Results The expression of miR-342-3p was transiently up-regulated in the early phase of OC generation and was down-regulated after 24-48 hour in OC precursors. While pre-miR-342$3 p$ promoted the motility of RAW264.7 cells, anti-miR-342-3p inhibited all motility parameters recorded $(p<0.0001$, ANOVA test). Anti-miR-342-3p reduced the proliferation $(p<0.01)$ and cell survival of OC precursors through a pro-apoptotic effect, as assessed by increased caspase $3-7$ activity $(p<0.01)$. Overall, miR-342-3p neutralization in OC precursors reduced OC numbers $(p<0.001)$ compared to the pre-miR-342-3p condition. In primary cells, miR-342-3p was up-regulated in bone marrowderived mature OC from arthritic mice compared to healthy controls ( $p=0.03$; STA $n=5 /$ group).

Conclusions Our data suggest that miR-342-3p promotes the early phase of osteoclastogenesis by enhancing the cell survival and motility of OC precursors. The up-regulation of miR-342$3 \mathrm{p}$ in OC isolated from arthritic mice may reflect the increased osteoclastogenic potential of inflammatory precursors in arthritis.

Disclosure of Interest None declared.

\section{P067 TOFACITINIB IMPAIRS MONOCYTE-DERIVED DENDRITIC CELL DIFFERENTIATION IN RHEUMATOID ARTHRITISAND PSORIATIC ARTHRITIS}

${ }^{1}$ V Marzaioli*, ${ }^{1} \mathrm{M}$ Canavan, ${ }^{1} \mathrm{~A}$ Floudas, ${ }^{1} \mathrm{~S}$ Wade, ${ }^{2} \mathrm{C}$ Low, ${ }^{2} \mathrm{DJ}$ Veale, ${ }^{1} \mathrm{U}$ Fearon. ${ }^{1} \mathrm{TBSI}$, Molecular Rheumatology, Trinity College Dublin; ${ }^{2}$ Centre for Arthritis and Rheumatic Diseases, St Vincent's University Hospital, UCD, Dublin, Ireland

\subsection{6/annrheumdis-2018-EWRR2019.56}

Career situation of first and presenting author Post-doctoral fellow.

Introduction Tofacitinib is an oral Janus kinase inhibitor, recently approved for the treatment of rheumatoid arthritis (RA) and psoriatic arthritis (PsA). Although its mechanism of action has been explored in circulating cells, including neutrophils and lymphocyte, its effect on dendritic cells development and function remains still to be elucidated. Monocyte-derived dendritic cells (Mo-DC) are a subset of inflammatory DC derived from circulating monocytes with a key role in inflammation and infection.

Objectives The aim of this project is to evaluate the effect of Tofacitinib on inflammatory Mo-DC differentiation from RA and PsA patients, an important step in innate immunity.

Methods Monocytes were isolated from blood of healthy donor (HC), RA and PsA patients by magnetic separation and differentiated in the presence of GM-CSF/IL-4 cocktail for 7 days. To evaluate the effect of Tofacitinib on Mo-DC differentiation, monocyte were pre-treated with $1 \mu \mathrm{M}$ Tofacitinib (or DMSO as control). CD209 (immature DC marker) was evaluated by flow cytometry in the $\mathrm{CD}_{11} \mathrm{c}^{+}$population. Non-specific macropinocytosis (using Lucifer Yellow) and receptormediated endocytosis (using $\mathrm{DQ}^{\mathrm{TM}}$ Ovalbumin) were investigated by flow cytometry. The effect of Tofacitinib on NADPH oxidases (NOX) 5 and 2 expression, known players in MoDC differentiation, was evaluated by Western blot analysis. Finally, the frequency of $\mathrm{CD} 209^{+}$cells and their chemokine receptor expression (CXCR3/4/5 and CCR6/7) were evaluated by flow cytometry in peripheral blood (PBMC), synovial fluid (SFMC) mononuclear cells and synovial tissue cell suspensions from RA and PsA patients.

Results Pre-treatment of Mo-DC with Tofacitinib inhibited Mo-DC differentiation in RA and PsA patients, as evident by reduced CD209 marker expression. The decreased ability of monocytes to differentiate into DC in the presence of Tofacitinib was translated into a functional impairment of endocytic ability, in particular in PsA patients, as observed by the decreased uptake of both $\mathrm{DQ}^{\mathrm{TM}}$ Ovalbumin and Lucifer Yellow. In addition, Tofacitinib decreased NOX5 and increased NOX2 protein expression in Mo-DC of PsA and RA Mo-DC, altering the NOX2/NOX5 balance. Finally, we identified $\mathrm{CD} 09^{+}$cells in the periphery of RA and PsA patients, which were enriched in SFMC and synovial tissue cell suspension, and presented with an increased expression of CCR7 and CXCR3/5.

Conclusions Together, these observations suggest a novel mechanism of action of Tofacitinib in RA and PsA, by inhibiting Mo-DC development, which may alter migration of DC to the joint and subsequent activation of the immune response.

Disclosure of Interest V. Marzaioli Grant/research support from: Pfizer, M. Canavan: None declared, A. Floudas : None declared, S. Wade : None declared, C. Low : None declared, D. Veale: None declared, U. Fearon Grant/research support from: Pfizer.

\section{P068 RHEUMATOID ARTHRITIS PERIPHERAL CD14+ MONOCYTES ARE HYPER-INFLAMMATORY, HYPER- GLYCOLYTIC AND RETAIN A MEMORY BIAS TOWARD M1 MACROPHAGES}

${ }^{1} \mathrm{~T}$ Mcgarry*, 'M Hanlon, ${ }^{1} \mathrm{U}$ Fearon, ${ }^{2} \mathrm{D}$ J Veale. ${ }^{1}$ Clinical Medicine, Trinity Biomedical Sciences Institute; ${ }^{2}$ Centre for Arthritis and Rheumatic Diseases, St. Vincent's University Hospital, Dublin, Ireland

\subsection{6/annrheumdis-2018-EWRR2019.57}

Career situation of first and presenting author Post-doctoral fellow.

Introduction Myeloid cells with a monocyte/macrophages phenotype are present in large numbers in the rheumatoid arthritis (RA) joint, significantly contributing to disease. 